\title{
TECNOLOGIAS EDUCACIONAIS NA PROMOÇÃO DA SAÚDE DO IDOSO
}

Andrea Márcia da Cunha Lima ${ }^{1}$

Carmem Silvia Laureano Dalle Piagge

Antônia Lêda Oliveira Silva ${ }^{1}$

Maria Lúcia do Carmo Cruz Robazzi ${ }^{1,2}$

Cláudia Batista Mélo

Selene Cordeiro Vasconcelos ${ }^{1}$ http://orcid.org/0000-0003-0152-3332

http://orcid.org/0000-0001-7999-2943

http://orcid.org/0000-0001-7758-2035

http://orcid.org/0000-0003-2364-5787

http://orcid.org/0000-0001-5300-3510

http://orcid.org/0000-0002-8828-1251

Objetivo: Identificar as evidências cientificas acerca das tecnologias educacionais na promoção da saúde do idoso. Método: Estudo de revisão integrativa, que utilizou as bases de dados Medline via PubMed, Web of Science, Scopus, Cinahl e Cochrane, no período de 2010 a 2019 , submetidos a uma categorização por temática. Resultados: Foram identificados 303 artigos nas bases pesquisadas e após leitura do título e resumo foram selecionados seis artigos para compor a amostra final. As tecnologias educacionais surgiram como ferramentas na promoção da saúde do idoso e qualificação de cuidadores. Os vídeos $(n=1)$, cartilhas $(n=1)$ e encontros grupais $(n=1)$ foram as tecnologias mais aceitas pelos idosos, no entanto os aplicativos para dispositivos móveis e computadores tiveram maior aceitação pelos cuidadores de idosos $(n=3)$. Além disso, os resultados destacaram que é necessário elaborar estratégias para integrar tecnologias educacionais inovadoras na assistência e em políticas públicas de saúde, ampliando seu alcance na promoção da saúde dos idosos. Conclusões: São diversas as tecnologias educacionais utilizadas na promoção da saúde do idoso. O conhecimento do perfil do público alvo é necessário para que a seleção da tecnologia educacional a ser empregada seja adequada e, desta forma, possa atingir sua finalidade, proporcionando autonomia e empoderamento dessa população

Descritores: Tecnologia Educacional; Promoção da Saúde; Idoso.

\section{EDUCATIONAL TECHNOLOGIES IN PROMOTION OF THE ELDERLY'S HEALTH}

Objective: To identify the scientific evidence about the educational technologies in health promotion for the elderly. Method: Integrative review study, which used the Medline databases via PubMed, Web of Science, Scopus, Cinahl and Cochrane, from 2010 to 2019 , submitted to a categorization by theme. Results: The search returned 303 articles on the databases and after reading the title and abstract, six articles were selected to compose the final sample. The educational technologies have emerged as tools in the health promotion of the elderly and qualification of caregivers. The videos ( $\mathrm{n}=1$ ), booklets ( $\mathrm{n}=1$ ) and group meetings ( $n=1$ ) were the most widely accepted technologies by the elderly, however the applications for mobile devices and computers had greater acceptance by caregivers of the elderly $(n=3)$. Moreover, the results highlighted the need for developing strategies to integrate innovative educational technologies in care and in public health policies, extending their scope in promoting the health of the elderly. Conclusions: There are various educational technologies used in the health promotion of the elderly. The knowledge of the profile of the target audience is necessary to ensure the selection of the adequate educational technology, and, in this way, to achieve its goal, providing autonomy and empowerment of this population.

Descriptors: Educational Technology; Health Promotion; Aged

\section{TECNOLOGÍAS EDUCATIVAS EN LA PROMOCIÓN DE LA SALUD DE LOS ANCIANOS}

Objetivo: Identificar la evidencia científica acerca de las tecnologías educativas en la promoción de la salud para los ancianos. Método: Estudio de revisión integral, que utilizó las bases de datos Medline a través de PubMed, Web of Science, Scopus, Cinahl y Cochrane, de 2010 a 2019 , sometido a una categorización por tema. Resultados: Se identificaron 303 artículos en las bases de datos y después de la lectura del título y del resumen, seis artículos fueron seleccionados para componer la muestra final. Las tecnologías educativas han surgido como herramientas en la promoción de la salud de los ancianos y la cualificación de los cuidadores. Los vídeos $(n=1)$, cartillas $(n=1)$ y reuniones de grupo $(n=1)$ fueron las tecnologías más ampliamente aceptada por los ancianos, sin embargo, las aplicaciones para dispositivos móviles y ordenadores tuvo mayor aceptación por parte de los cuidadores de pacientes ancianos ( $\mathrm{n}=3$ ). Además, los resultados destacaron que existe una necesidad de desarrollar estrategias para integrar tecnologias educativas innovadoras en la atención y en las políticas de salud pública, ampliando su alcance en la promoción de la salud de los ancianos. Conclusiones: Existen diversas tecnologías educativas utilizadas en la promoción de la salud en los ancianos. El conocimiento del perfil del público es necesario para garantizar la selección de la tecnología educativa adecuada, y de esta manera, lograr su objetivo, proporcionando la autonomía y el empoderamiento de esta población

Descriptores: Tecnologia Educativa; Promoción de la Salud: Anciano.

${ }^{1}$ Universidade Federal da Paraiba, PB, Brasil

${ }^{2}$ Escola de Enfermagem de Ribeirão Preto da Universidade de São Paulo, SP, Brasil.

Autor Correspondente: Andrea Márcia da Cunha Lima - Email: andrealima2006@gmail.com

Recebido: 06/03/2020 - Aceito: 17/07/2020 


\section{INTRODUÇÃO}

A população mundial tem envelhecido em um ritmo acelerado e o Brasil não é exceção. A expectativa de vida da população está aumentando, as taxas de natalidade diminuindo e o país está se transformando em uma sociedade envelhecida ${ }^{1}$.

Segundo o Instituto Brasileiro de Geografia e Estatística (IBGE)², em 2018, existiam 28 milhões de brasileiros idosos, o que representava pouco mais de $13,4 \%$ da população. As projeções populacionais indicam que, em 2035, o grupo com 60 anos ou mais representará $20,9 \%$ da população, alcançando 30,4\% em 2055.

O envelhecimento populacional representa um grande desafio tanto para a sociedade como para os órgãos governamentais. Isso tem sido foco de estudos na Gerontologia e nas Ciências Humanas e Sociais ${ }^{3}$.

De acordo com as diretrizes da Política Nacional de Saúde da Pessoa Idosa, Lei 2.528, não se fica velho aos 60 anos. $O$ envelhecimento é um processo natural que ocorre ao longo de toda a experiência de vida do ser humano, por meio de escolhas e de circunstâncias ${ }^{4}$. A referida Política considera, também, que se deve envelhecer com saúde, de forma ativa, livre de qualquer tipo de dependência funcional e isso exige promoção da saúde em todas as idades ${ }^{3}$.

Nesse âmbito, cabe à educação em saúde promover hábitos de vida saudáveis ao articular saberes técnicos e populares e mobilizar recursos individuais e coletivos. $\bigcirc$ trabalho coletivo incita a elaboração de programas educacionais para a promoção da saúde voltados à população idosa ${ }^{5}$

O termo "Promoção da Saúde" configura-se como um conjunto de estratégias e formas de produzir saúde, tanto no âmbito individual como no coletivo, no intuito de atender às necessidades sociais de saúde e garantir a melhoria da qualidade de vida da população ${ }^{6}$.

Um dos principais dispositivos para promover o cuidado da saúde é a educação em saúde, com um caráter mais ampliado, que auxilia não somente na prevenção de doenças, mas também, no desenvolvimento da responsabilidade individual, sendo bastante utilizada para a transformação de práticas e comportamentos individuais, além do desenvolvimento da autonomia e da qualidade de vida do indivíduo ${ }^{7}$.

Nesse contexto, as tecnologias educativas mostram-se efetivas para promover a saúde, pois aperfeiçoam o conhecimento e o enfrentamento do paciente, tornando-o capaz de entender como as próprias ações influenciam a sua condição de saúde ${ }^{8}$.

Entende-se a palavra "tecnologia" como o modo de fazer, o método e não, apenas, a tecnologia física em si, como máquinas e equipamentos de ponta. As tecnologias em saúde são divididas em três perspectivas: leves, que são as utilizadas nas relações humanas; leve-duras que se referem aos saberes profissionais, bem estruturados com a clínica, a epidemiologia e os demais profissionais que compõem a equipe e as duras que se referem ao instrumental complexo em seu conjunto, englobando todos os equipamentos para tratamentos, exames e a organização das informações?.

Do exposto, o estudo tem como objetivo identificar as evidências científicas acerca das tecnologias educacionais na promoção da saúde do idoso.

\section{MÉTODO}

Este estudo trata-se de uma Revisão Integrativa da Literatura-RIL, com a finalidade de compilar os resultados de estudos publicados sobre a temática, contribuindo para o acesso ao conhecimento científico e para as tomadas de decisões dos profissionais, baseadas em evidências científicas.

Há estudos científicos disponiveis que descrevem as etapas de preparação para a Revisão Integrativa, contudo, podem haver diferenças de conceituação e descrição das etapas entre os autores. Para sua a construção, sugere-se seguir o cumprimento das seguintes fases, as quais foram implementadas no presente estudo: elaboração da pergunta norteadora; busca dos artigos nas bases de dados indexadas; coleta de dados; leitura e avaliação dos artigos selecionados; discussão dos resultados; sintese do conhecimento e apresentação da revisão integrativa ${ }^{10}$.

Identificado e selecionado o tema, consolidou-se a pergunta norteadora: Quais as tecnologias educacionais que são utilizadas na promoção da saúde do idoso?, seguindo a estratégia PICo (População, Interesse e Contexto). Neste estudo, a população foi representada por idosos; o interesse configurou-se pela tecnologia educacional e o contexto caracterizado pela promoção da saúde.

Para a busca na literatura, foram definidas cinco bases de dados: Medical Literature Analysis and Retrieval System Online (MEDLINE via PubMed), Web of Science, Scopus, Cumulative Index to Nursing and Allied Health Literature (CINAHL) e Cochrane Library. Também, foram utilizados os descritores indexados nos Descritores em Ciência da Saúde (DeCS) e no Medical Subject Headings (Mesh Terms) - "educational technology" AND "health promotion" AND aged. A estratégia de busca seguiu as especificidades de cada base, mantendo o atendimento à pergunta norteadora. 
Quadro 1. Descritores controlados empregados na estratégia de busca sobre promoção da saúde em idosos por meio de tecnologias educacionais, para população, interesse e contexto, e histórico da busca em cada base de dados, 2019.

\begin{tabular}{|c|c|}
\hline \multicolumn{2}{|c|}{ MEDLINE via PubMed } \\
\hline$P$ & aged \\
\hline I & educational technology \\
\hline Co & health promotion \\
\hline $\begin{array}{l}\text { Histórico da } \\
\text { busca }\end{array}$ & $\begin{array}{l}\text { "educational technology" AND "health promotion" AND } \\
\text { aged }\end{array}$ \\
\hline \multicolumn{2}{|c|}{ Web of Science } \\
\hline$P$ & aged \\
\hline I & educational technology \\
\hline Co & health promotion \\
\hline $\begin{array}{l}\text { Histórico da } \\
\text { busca }\end{array}$ & $\begin{array}{l}\text { "educational technology" AND "health promotion" AND } \\
\text { aged }\end{array}$ \\
\hline \multicolumn{2}{|l|}{ Scopus } \\
\hline$P$ & aged \\
\hline I & educational technology \\
\hline Co & health promotion \\
\hline $\begin{array}{l}\text { Histórico da } \\
\text { busca }\end{array}$ & $\begin{array}{l}\text { "educational technology" AND "health promotion" AND } \\
\text { aged }\end{array}$ \\
\hline \multicolumn{2}{|l|}{ CINAHL } \\
\hline$P$ & aged \\
\hline I & educational technology \\
\hline Co & health promotion \\
\hline $\begin{array}{l}\text { Histórico da } \\
\text { busca }\end{array}$ & $\begin{array}{l}\text { "educational technology" AND "health promotion" AND } \\
\text { aged }\end{array}$ \\
\hline \multicolumn{2}{|l|}{ Cochrane } \\
\hline$P$ & aged \\
\hline I & educational technology \\
\hline Co & health promotion \\
\hline $\begin{array}{l}\text { Histórico da } \\
\text { busca }\end{array}$ & $\begin{array}{l}\text { "educational technology" AND "health promotion" AND } \\
\text { aged }\end{array}$ \\
\hline
\end{tabular}

Os critérios de inclusão estabelecidos foram: artigos primários publicados entre os anos de 2010 a 2019, com abordagem da temática das tecnologias educacionais para a promoção da saúde do idoso, com indexação em, pelo menos, uma das bases de dados referenciadas. Como critérios de exclusão foram considerados as publicações repetidas nas bases de dados; estudos de caso; relato de experiência; teses; dissertações; artigos de opinião e outros artigos de revisão.

Foram identificados 303 artigos e após a aferição da relação entre os títulos e a resposta à pergunta norteadora, obteve-se 29 potencialmente elegíveis, sendo excluído 1 por estar duplicado nas bases de dados, resultando em 28. Após a leitura dos resumos e com a aplicação dos demais critérios estabelecidos, foram excluídos mais 22 , res- tando na amostra final 6 artigos na revisão. Para análise e extração dos dados dos artigos selecionados, utilizou-se o instrumento previamente elaborado e testado ${ }^{11}$, adaptado para este estudo. Os resultados foram organizados por meio de uma síntese descritiva com dados quantitativos e qualitativos. Como diretrizes metodológicas para composição do relatório de Revisão foi utilizado o checklist PRISMA (Principais Itens para Relatar Revisões Sistemáticas e Meta-análises) (Figura 1).

Figura 1. Processo de seleção amostral dos artigos identificados sobre tecnologias educacionais na promoção da saúde do idoso. João Pessoa - PB, 2010-2019.

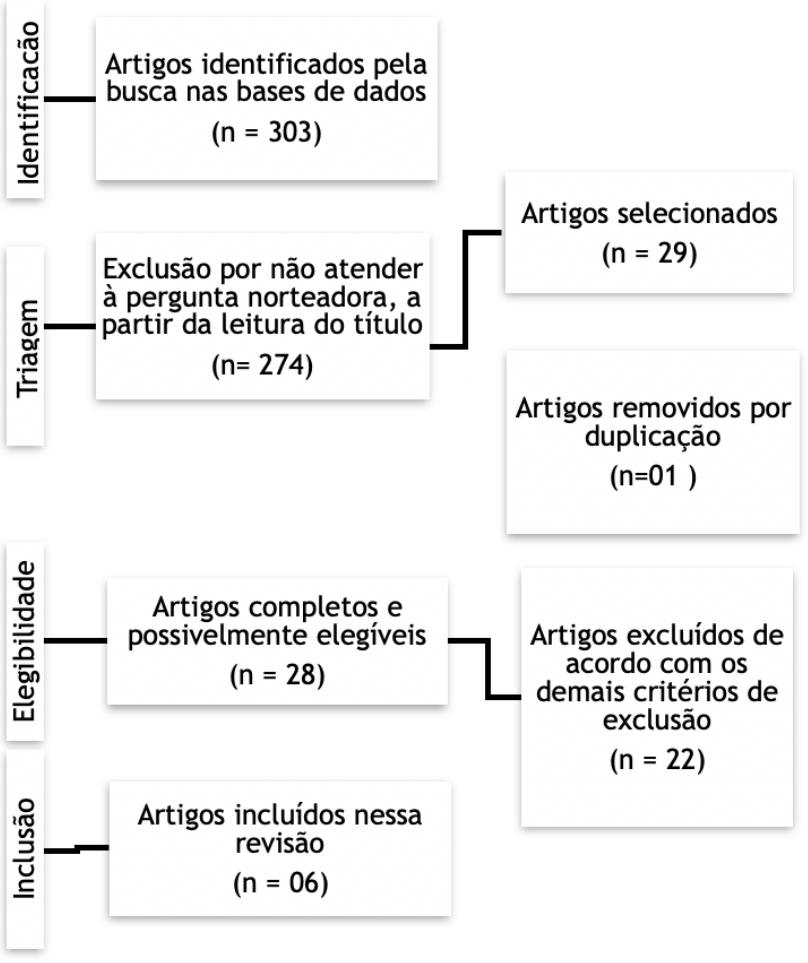

No processo de categorização dos artigos selecionados, emergiram quatro categorias de tecnologias educacionais para promoção da saúde do idoso: I- vídeos educativos, II- cartilhas, III- encontros grupais e IV- aplicativos para dispositivos móveis e computadores.

Em relação à classificação dos níveis de evidência, considerou-se: Nível 1 - evidências resultantes da meta-análise de múltiplos estudos clínicos controlados e randomizados; Nivel 2 - evidências obtidas em estudos individuais com delineamento experimental; Nível 3 - evidências de estudos quase-experimentais; Nivel 4 - evidências de estudos descritivos (não-experimentais) ou com abordagem qualitativa; Nivel 5 - evidências provenientes de relatos de caso ou de experiência; e Nível 6 - evidências baseadas em opiniões de especialistas ${ }^{12}$. 


\section{RESULTADOS}

Essa Revisão Integrativa mostrou que as tecnologias educacionais abordaram aspectos relacionados à assistência e ao autocuidado de idosos, provenientes da construção de um saber técnico-científico resultante de investigações, aplicações de teorias e da experiência cotidiana dos profissionais com os usuários, que visaram facilitar processos de ensino-aprendizagem.

Da amostra final de seis artigos, três foram de autores brasileiros, publicados nas revistas: Journal of Nursing UFPE/Revista de Enfermagem UFPE, Enfermagem em Foco e Revista Gaúcha de Enfermagem e três de autores estrangeiros (Austrália, Portugal e Taiwan), publicados nos periódicos: International Psychogeriatrics, Revista Latino-Americana de Enfermagem e Computers in Human Behavior. Salienta-se que apenas um artigo foi publicado em revista especializada no cuidado ao idoso.

Os artigos foram sumarizados a partir de suas características gerais em um quadro-sintese para registro dos dados, de maneira a organizar as informações obtidas segundo os elementos disponiveis na sua identificação.

Quadro 2. Caracterização dos artigos selecionados sobre tecnologias educacionais na promoção da saúde do idoso. João Pessoa - PB, 2010-2019.

\begin{tabular}{|c|c|c|c|c|c|}
\hline Título & $\begin{array}{l}\text { Autor/ } \\
\text { Ano/ } \\
\text { Pais }\end{array}$ & $\begin{array}{l}\text { Tipo de } \\
\text { estudo }\end{array}$ & Objetivo & $\begin{array}{l}\text { Resulta- } \\
\text { dos prin- } \\
\text { cipais }\end{array}$ & $\begin{array}{l}\text { Nivel de } \\
\text { Evidên- } \\
\text { cia / } \\
\text { Cate- } \\
\text { gorias } \\
\text { Identi- } \\
\text { ficadas }\end{array}$ \\
\hline $\begin{array}{l}\text { Sexuality } \\
\text { and aging: } \\
\text { identified } \\
\text { needs for } \\
\text { construc- } \\
\text { tion of an } \\
\text { educa- } \\
\text { tional } \\
\text { technol- } \\
\text { ogy }\end{array}$ & $\begin{array}{l}\text { Araújo; Mo- } \\
\text { reira; Silva, } \\
\text { Aragão, } \\
\text { Freitas; } \\
\text { Monteiro } \\
2017 \text {. } \\
\text { Brasil }^{13}\end{array}$ & $\begin{array}{l}\text { Qualitati- } \\
\text { vo Explo- } \\
\text { ratório }\end{array}$ & $\begin{array}{l}\text { Identificar } \\
\text { as neces- } \\
\text { sidades } \\
\text { dos idosos } \\
\text { diante } \\
\text { de sua } \\
\text { sexuali- } \\
\text { dade para } \\
\text { subsidiar a } \\
\text { cons- } \\
\text { trução } \\
\text { de uma } \\
\text { tecnologia } \\
\text { educativa }\end{array}$ & $\begin{array}{l}\text { Os recur- } \\
\text { sos de } \\
\text { video e/ } \\
\text { ou visuais } \\
\text { utili- } \\
\text { zados, } \\
\text { aliados à } \\
\text { interação } \\
\text { profis- } \\
\text { sional, } \\
\text { foram } \\
\text { identi- } \\
\text { ficados } \\
\text { como as } \\
\text { tecnolo- } \\
\text { gias mais } \\
\text { ade- } \\
\text { quadas } \\
\text { capazes } \\
\text { de pro- } \\
\text { mover a } \\
\text { compre- } \\
\text { ensão do } \\
\text { assunto } \\
\text { pelos } \\
\text { idosos }\end{array}$ & $\begin{array}{l}4 \\
\text { I }\end{array}$ \\
\hline
\end{tabular}

\begin{tabular}{|c|c|c|c|c|c|}
\hline $\begin{array}{l}\text { Per- } \\
\text { son-cen- } \\
\text { tered } \\
\text { care and } \\
\text { engage- } \\
\text { ment via } \\
\text { technol- } \\
\text { ogy of } \\
\text { residents } \\
\text { with de- } \\
\text { mentia in } \\
\text { aged care } \\
\text { facilities }\end{array}$ & $\begin{array}{l}\text { Goh; Loi; } \\
\text { Westphal; } \\
\text { Lauten- } \\
\text { schlager } \\
\text { 2017, } \\
\text { Australiaa }^{14}\end{array}$ & $\begin{array}{l}\text { Transver- } \\
\text { sal }\end{array}$ & $\begin{array}{l}\text { Fornecer } \\
\text { educação } \\
\text { e explorar } \\
\text { as opi- } \\
\text { niões e } \\
\text { barreiras } \\
\text { pelos } \\
\text { cuidado- } \\
\text { res sobre } \\
\text { o uso da } \\
\text { tecno- } \\
\text { logia de } \\
\text { toque na } \\
\text { tela para } \\
\text { envolver } \\
\text { seus } \\
\text { residen- } \\
\text { tes com } \\
\text { demência }\end{array}$ & $\begin{array}{l}\text { O uso de } \\
\text { tecnolo- } \\
\text { gias no } \\
\text { cuidado } \\
\text { de resi- } \\
\text { dentes } \\
\text { com } \\
\text { demência } \\
\text { demons- } \\
\text { trou ser } \\
\text { uma } \\
\text { estraté- } \\
\text { gia que } \\
\text { ampliou a } \\
\text { confian- } \\
\text { ça e o } \\
\text { conhe- } \\
\text { cimento } \\
\text { dos } \\
\text { cuidado- } \\
\text { res e a } \\
\text { melhora } \\
\text { no aten- } \\
\text { dimento }\end{array}$ & IV \\
\hline $\begin{array}{l}\text { Evalua- } \\
\text { tion of the } \\
\text { educa- } \\
\text { tional } \\
\text { tech- } \\
\text { nology } \\
\text { "Caring } \\
\text { for de- } \\
\text { pendent } \\
\text { people" } \\
\text { by family } \\
\text { caregivers } \\
\text { in chang- } \\
\text { es and } \\
\text { trans- } \\
\text { fers of } \\
\text { patients } \\
\text { and tube } \\
\text { feeding }\end{array}$ & $\begin{array}{l}\text { Landeiro; } \\
\text { Peres; } \\
\text { Martins } \\
\text { 2016, } \\
\text { Portugal }{ }^{15}\end{array}$ & $\begin{array}{l}\text { Experi- } \\
\text { mental } \\
\text { não } \\
\text { Randomi- } \\
\text { zado }\end{array}$ & $\begin{array}{l}\text { Avaliar as } \\
\text { contribui- } \\
\text { ções da } \\
\text { tecnologia } \\
\text { interativa } \\
\text { educacio- } \\
\text { nal no de- } \\
\text { senvolvi- } \\
\text { mento do } \\
\text { conheci- } \\
\text { mento dos } \\
\text { cuidado- } \\
\text { res fami- } \\
\text { liares no } \\
\text { domicilio e } \\
\text { avaliar sua } \\
\text { satisfação } \\
\text { com o seu } \\
\text { uso }\end{array}$ & $\begin{array}{l}\text { A tec- } \\
\text { nologia } \\
\text { educa- } \\
\text { cional } \\
\text { interativa } \\
\text { empre- } \\
\text { gada } \\
\text { (aplicati- } \\
\text { vo para } \\
\text { compu- } \\
\text { tador) } \\
\text { atendeu } \\
\text { com } \\
\text { sucesso a } \\
\text { qualidade } \\
\text { técnica } \\
\text { e as } \\
\text { necessi- } \\
\text { dades de } \\
\text { aprendi- } \\
\text { zado dos } \\
\text { cuidado- } \\
\text { res e foi } \\
\text { conside- } \\
\text { rada }\end{array}$ & IV \\
\hline $\begin{array}{l}\text { Cultivo } \\
\text { do bem } \\
\text { viver das } \\
\text { pessoas } \\
\text { idosase } \\
\text { tecno- } \\
\text { logia } \\
\text { cuidativo } \\
\text {-educa- } \\
\text { cional de } \\
\text { enferma- } \\
\text { gem }\end{array}$ & $\begin{array}{l}\text { Goes; } \\
\text { Polaro; } \\
\text { Gonçalves } \\
2016 \text {, } \\
\text { Brasil }^{16}\end{array}$ & $\begin{array}{l}\text { Quali- } \\
\text { tativo e } \\
\text { Quantita- } \\
\text { tivo }\end{array}$ & $\begin{array}{l}\text { Realizar } \\
\text { avaliação } \\
\text { diagnós- } \\
\text { tica de } \\
\text { condições } \\
\text { de vida e } \\
\text { saúde dos } \\
\text { idosose } \\
\text { testar o } \\
\text { desenvol- } \\
\text { vimento } \\
\text { de uma } \\
\text { tecnologia } \\
\text { cuidativo- } \\
\text {-educa- } \\
\text { cional }\end{array}$ & $\begin{array}{l}\text { O enfer- } \\
\text { meiro } \\
\text { atuou } \\
\text { como } \\
\text { facilita- } \\
\text { dor no } \\
\text { desenvol- } \\
\text { vimento } \\
\text { de com- } \\
\text { petências } \\
\text { para o } \\
\text { autocui- } \\
\text { dado de } \\
\text { idosos, } \\
\text { instru- } \\
\text { mentali- } \\
\text { zado por } \\
\text { diretrizes } \\
\text { de tecno- } \\
\text { logia leve } \\
\text { (encon- } \\
\text { tros } \\
\text { grupais) } \\
\text { de atos } \\
\text { de rela- } \\
\text { ção no } \\
\text { cuidado }\end{array}$ & III \\
\hline
\end{tabular}




\begin{tabular}{|c|c|c|c|c|c|}
\hline $\begin{array}{l}\text { The } \\
\text { attitudes, } \\
\text { impact, } \\
\text { and learn- } \\
\text { ing needs } \\
\text { of older } \\
\text { adults } \\
\text { using } \\
\text { apps on } \\
\text { touch- } \\
\text { screen } \\
\text { mobile } \\
\text { devices: } \\
\text { Results } \\
\text { from a pi- } \\
\text { lot study }\end{array}$ & $\begin{array}{l}\text { Chiu; Hu; } \\
\text { Lin; Chang; } \\
\text { Chang; Lai } \\
\text { 2016, } \\
\text { Taiwan }^{17}\end{array}$ & $\begin{array}{l}\text { Qualita- } \\
\text { tivo }\end{array}$ & $\begin{array}{l}\text { Compre- } \\
\text { ender a } \\
\text { aceitação } \\
\text { e limita- } \\
\text { ções da } \\
\text { tecno- } \\
\text { logia da } \\
\text { Internet } \\
\text { por parte } \\
\text { dos adul- } \\
\text { tos mais } \\
\text { velhos }\end{array}$ & $\begin{array}{l}\text { Os dis- } \\
\text { positivos } \\
\text { móveis } \\
\text { com telas } \\
\text { sensiveis } \\
\text { ao toque, } \\
\text { em apli- } \\
\text { cativos } \\
\text { direcio- } \\
\text { nados à } \\
\text { saúde, } \\
\text { entrete- } \\
\text { nimento, } \\
\text { trans- } \\
\text { porte e } \\
\text { midias } \\
\text { sociais } \\
\text { contri- } \\
\text { buiu para } \\
\text { o bem } \\
\text { estar } \\
\text { psicoló- } \\
\text { gico em } \\
\text { idosos, } \\
\text { mesmo }\end{array}$ & $\begin{array}{l}4 \\
\text { IV }\end{array}$ \\
\hline $\begin{array}{l}\text { Geron- } \\
\text { totec- } \\
\text { nologia } \\
\text { educativa } \\
\text { voltada } \\
\text { ao idoso } \\
\text { estomi- } \\
\text { zado à } \\
\text { luz da } \\
\text { complexi- } \\
\text { dade }\end{array}$ & $\begin{array}{l}\text { Barros; } \\
\text { Santos; } \\
\text { Gomes; } \\
\text { Erdmann } \\
2012 \text {; } \\
\text { Brasil }^{18}\end{array}$ & $\begin{array}{l}\text { Qualita- } \\
\text { tivo }\end{array}$ & $\begin{array}{l}\text { Apre- } \\
\text { sentar a } \\
\text { cartilha } \\
\text { educativa } \\
\text { como um } \\
\text { produto } \\
\text { geronto- } \\
\text { tecnoló- } \\
\text { gico útil } \\
\text { para o } \\
\text { cuidado } \\
\text { ao idoso } \\
\text { estomi- } \\
\text { zado }\end{array}$ & $\begin{array}{l}\text { A tec- } \\
\text { nologia } \\
\text { educativa } \\
\text { do tipo } \\
\text { cartilha } \\
\text { consti- } \\
\text { tuiu-se } \\
\text { como } \\
\text { uma } \\
\text { forma de } \\
\text { cuidado } \\
\text { huma- } \\
\text { nizado } \\
\text { promotor } \\
\text { da eman- } \\
\text { cipação } \\
\text { do idoso } \\
\text { estomi- } \\
\text { zado }\end{array}$ & $\begin{array}{l}4 \\
\text { II }\end{array}$ \\
\hline
\end{tabular}

\section{DISCUSSÃO}

A elaboração de tecnologias educacionais exige procedimentos metodológicos rigorosos, corroborando com os resultados dessa Revisão que mostrou desde a escolha de teorias e temáticas relacionadas à assistência até o autocuidado de idosos. De uma maneira geral, essas tecnologias possibilitam a difusão de conhecimentos e podem provocar mudanças, favorecendo ações que influenciam no padrão de saúde das pessoas ${ }^{19}$.

O uso das tecnologias vem crescendo, consideravelmente, ao longo dos anos, trazendo grandes possibilidades de utilização de novos recursos nas práticas de cuidado e ensino de saúde ${ }^{20}$, como observado no presente estudo que mostrou diversos tipos de tecnologias educacionais, com destaque para vídeos, aplicativos para dispositivos móveis, cartilhas e/ou manuais, além do uso de tecnologia leve, por meio de encontros grupais com abordagem de temas relacionados à saúde.

Dentre esses recursos, o vídeo educativo apresenta-se como um instrumento didático e tecnológico, constituin- do-se uma ferramenta que proporciona conhecimento, favorece a consciência crítica e a promoção da saúde ${ }^{21}$. Por ser uma estratégia audiovisual exerce maior impacto na aprendizagem quando comparada com a linguagem escrita, pois possui uma série de imagens com movimentos gráficos, textos e sons que permitem ao espectador melhor compreensão do tema apresentado, facilitando a construção do conhecimento, principalmente se possuir até 10 minutos de duração ${ }^{22-23}$. E quando aliados à interação profissional, é identificado como a tecnologia mais apropriada capaz de promover a compreensão do assunto pelos idosos ${ }^{13}$.

Nesse contexto, novas tecnologias vão surgindo $e$ favorecendo as estratégias de comunicação, como os smartphones que têm se popularizado nos últimos anos. Equipados com câmeras digitais e aplicativos, tornam-se ferramentas de qualidade para a ampliação do acesso à informação, além de aproximar os profissionais ao idoso, proporcionando uma maior rede de apoio, por meio do diálogo, troca de experiências e dúvidas no tocante aos seus cuidados. Esta abordagem ainda oferece praticidade e baixo custo, atingindo populações com altos niveis de necessidade e que não têm acesso às orientações sobre os cuidados $^{24-25}$.

O uso de tecnologia educacional, por meio de aplicativos, apresenta-se como uma estratégia para o desenvolvimento do conhecimento em cuidadores de idosos nos diferentes domínios de autocuidado, além de ampliar a confiança e proporcionar melhoria no atendimento à essas pessoas $^{14-15}$.

Os dispositivos móveis com telas sensiveis ao toque, em aplicativos direcionados à saúde, entretenimento. transporte e mídias sociais contribuem para o bem estar psicológico em idosos. No entanto, esses indivíduos podem apresentar dificuldades para aprender a usar a internet, necessitando de assistência técnica de familiares e amigos o que pode favorecer, efetivamente, a melhoria de sua motivação para a adoção da internet e de suas aplicações ${ }^{17}$.

A terceira idade ainda precisa ser mais inserida nesse universo informatizado ${ }^{26}$, fato evidenciado por alguns dos estudos nessa Revisão, que mostraram as dificuldades de acesso e falta de habilidade de manuseio dos recursos eletrônicos e tecnológicos como forma de promoção da saúde, tornando-os menos aceitos pelos idosos ${ }^{14-15,17}$.

Entretanto, à medida que as informações e os serviços estão se tornando cada vez mais descentralizados e disponíveis, espera-se que um número crescente de idosos use serviços baseados na internet - saúde, educação, finanças e outros. Nesse sentido, estudo realizado no México, que 
objetivou mostrar um workshop com base no sistema de gerenciamento de aprendizagem como uma ferramenta de apoio à alfabetização digital de idosos, mostrou que essas pessoas são capazes de aprender e adquirir habilidades de alfabetização digital, desde que estejam fortemente motivados ou conheçam os benefícios funcionais relacionados às Tecnologias de Informação e Comunicação ${ }^{27}$.

A abordagem participativa, comunicativa e coletiva tem sido utilizada para promover a inserção dos idosos nessa realidade digital, sendo fundamental no desenvolvimento de tecnologias educacionais, na interação e na troca de conhecimentos.

Por meio de encontros grupais, o enfermeiro é um agente facilitador no desenvolvimento de competências para o autocuidado e estimulador dos idosos como protagonistas do próprio envelhecimento ${ }^{16}$.

Corroborando com os resultados encontrados, as tecnologias educacionais leves foram as mais utilizadas para idosos como grupos terapêuticos, rodas de conversa, dinâmicas, palestras, cartilhas e panfletos ${ }^{28}$.

A estratégia do trabalho em grupo é vantajosa para as instituições de saúde. A dinâmica de grupo com idosos potencializou seu empoderamento e foi a mais desejada por eles, somando-se as palestras, conversas, panfletos informativos, entre outros ${ }^{29}$.

A cartilha apresenta-se como uma gerontotecnologia capaz de facilitar a compreensão do idoso sobre diferentes temáticas de saúde ${ }^{30}$, com alcance na educação da família ou outro grupo de apoio para o cuidado ${ }^{18,31}$. Por outro lado, pode ser ferramenta inviável para idosos de baixa escolaridade, pela incompreensão do conteúdo do material, pois ainda existe um significativo número de pessoas idosas que apresentam algum grau de dificuldade para a leitura, sendo um ponto negativo de tais tecnologias impressas ${ }^{28,32}$. Entretanto, ainda não se identificou algum estudo que avaliasse, comparativamente, a efetividade do uso de cartilhas ou outro material impresso com aqueles produzidos digitalmente, para uso na promoção de saúde dos idosos.

Como identificado nos artigos desta revisão, as cartilhas e os demais materiais educativos assumem um papel importante no processo da educação em saúde, pois além de facilitarem a divulgação de conteúdos de aprendizagem, funcionam como um recurso que pode ser disponibilizado para o paciente e sua família ${ }^{33}$. Somado a isso, auxiliam para que a educação seja um processo contínuo, como um material de constante pesquisa, em caso de dúvidas e angústia sobre o cuidado ${ }^{34}$.

Esta revisão evidencia que há concordância entre os autores em relação às informações e à linguagem dos diver- sos materiais empregados nas tecnologias educacionais, devendo ser de fácil compreensão e adequada ao público alvo. Materiais desenvolvidos para fins educativos devem obedecer a uma linguagem simples, com menor nível de leitura, que possibilite transmitir informações precisas. Aliado à função de informar, devem estimular a reflexão e preparar o leitor para o cuidado ${ }^{34-35}$.

Apesar da diversidade de tecnologias educacionais empregadas na promoção da saúde, não foi identificado nessa revisão, o recurso dos infográficos, que são textos visuais explicativos e informativos associados aos elementos como imagens, sons, gráficos, entre outros. Essa linguagem, que dá preferência à imagem, parece encaixar-se mais adequadamente ao estilo de vida da população. O infográfico é lido em poucos minutos, já que é predominantemente visual e de fácil compreensão, a fim de atingir um número maior de leitores ${ }^{36}$. Por possibilitar a combinação de diversos recursos multimídia, os infográficos podem unir os benefícios áudio visuais e tornarem-se potenciais ferramentas gerontotecnológicas educacionais.

Sendo assim, é de fundamental importância que as tecnologias educativas gerontogeriátricas sejam amplamente discutidas no cenário da saúde, tanto entre os profissionais quanto entre a população idosa e que novas tecnologias sejam desenvolvidas em prol da assistência aos indivíduos, como forma de inovação, capaz de produzir mudança no processo de envelhecer, principalmente com foco no empoderamento, autonomia e qualidade de vida nessa faixa etária ${ }^{28}$

\section{Limitações do estudo}

Essas limitações relacionam-se com a escassez de publicações sobre a temática abordada, além de estudos com baixo nível de evidência o que pode comprometer a sumarização dos resultados.

\section{Contribuições para prática}

A sintese dos resultados identificados nessa revisão aponta para importantes contribuições para a prática do cuidado integral na promoção da saúde do idoso, por promover reflexões acerca da amplitude e diversidade do uso de tecnologias educacionais, direcionadas para o incentivo de mudanças comportamentais dessa população, relacionadas aos cuidados de saúde e bem-estar.

\section{Contribuição dos autores:}

Contribuição intelectual na revisão crítica do conteúdo e aprovação da versão final para publicação. 


\section{CONCLUSÃO}

O estudo possibilitou ampliar o conhecimento e identificar as tecnologias educacionais para a promoção da saúde de idosos, mostrando a diversidade de tipos, abordagens e temáticas utilizadas, não se restringindo apenas às tecnologias por meio de equipamentos e/ou aparelhos. As tecnologias leves, leves-dura e dura foram aceitas e utilizadas pelos cuidadores, porém as leves e leves-dura foram as mais aceitas entre os idosos, por proporcionarem interação profissional-idoso.
Faz-se necessário elaborar estratégias para integrar tecnologias educacionais inovadoras na assistência e em políticas públicas de saúde, ampliando seu alcance na promoção da saúde dos idosos. O conhecimento do perfil do público alvo aliado à linguagem acessível e de fácil compreensão são fundamentais para que a tecnologia educacional seja adequada e, desta forma possa atingir sua finalidade, proporcionando autonomia e empoderamento dessa população.

\section{REFERÊNCIAS}

1. Fernández-Ardèvol M. Práticas digitais móveis das pessoas idosas no Brasil. Panorama Setorial [Internet]. 2019: 11(1): 1-20. Disponivel em: https://www.cetic.br/media/docs/publicacoes/1/panorama_estendido_ mar_2019_online.pdf. Acesso em: 23 mar 2019.

2. Instituto Brasileiro de Geografia e Estatistica (IBGE). Tabelas 2018 - Projeções da População do Brasil e Unidades da Federação por sexo e idade: 2010-2060 [Internet]. Disponivel em: https://www.ibge. gov.br/estatisticas-novoportal/sociais/populacao/9109-projecao-da-populacao.html?=\&t=resultados. Acesso em: 5 dez. 2018

3. Ferreira MMM. A velhice: olhares das pessoas idosas e seus familiares. 2019. 174 f. Tese (Doutorado em Familia na Sociedade Contemporânea) Universidade Católica de Salvador, Salvador-BA, 2019. Disponivel em: http:// ri.ucsal.br:8080/jspui/bitstream/prefix/852/1/TESEMARILAINEFERREIRA. pdf. Acesso em: 17 set 2019

4. Brasil. Lei Federal no 2.528, de 19 de outubro de 2006. Política Nacional de Saúde da Pessoa Idosa [Internet]. Disponivel em: http://bvsms.saude. gov.br/bvs/saudelegis/gm/2006/prt2528_19_10_2006.html. Acesso em: 17 jul 2019.

5. Mallmann DG, Galindo Neto NM, Sousa JDC, Vasconcelos EMRD. Educação em saúde como principal alternativa para promover a saúde do idoso. Ciência Saúde Coletiva [Internet]. 2015; 20(6): 1763-72. 2015. Disponivel em : https://www.scielosp.org/pdf/csc/2015.v20n6/1763-1772/pt. Acesso em: 11 jun 2019.

6. Malta DC, Morais Neto O, Silva MMAD, Rocha D, Castro AMD, Reis AACD et al. Política Nacional de Promoção da Saúde (PNPS): capitulos de uma caminhada ainda em construção. Ciência \& Saúde Coletiva [Internet]. 2016 21(6): 1683-94. Disponivel em: https://www.scielosp.org/pdf/csc/2016. v2ln6/1683-1694/pt. Acesso em: 17 set 2019.

7. Janini JP, Bessler D, Vargas AB. Educação em saúde e promoção da saúde: impacto na qualidade de vida do idoso. Saúde em Debate [Internet]. 2015; 39:480-90. Disponivel em : https://www.scielosp.org/article/ sdeb/2015.v39n105/480-490/pt/. Acesso em: 17 set 2019.

8. Silveira MS, Cogo ALP. Contribuições das tecnologias educacionais digitais no ensino de habilidades de enfermagem: revisão integrativa. Revista gaúcha de enfermagem [Internet]. 2017; 38(2): e66204. Disponivel em:

http://www.scielo.br/pdf/rgenf/v38n2/0102-6933-rgenf-1983-144720170266204.pdf. Acesso em: 15 abr 2019.

9. Cestari VRF, Sampaio LRL, Barbosa IV, Studart RMB., Moura BBF, Araújo AR. Tecnologias do Cuidado utilizadas pela Enfermagem na Assistência ao
Paciente Politraumatizado: Uma Revisão Integrativa. Cogitare Enfermagem [Internet]. 2015; 20(4): 701-10. Disponivel em:

https://www.redalyc.org/pdf/4836/483647681022.pdf. Acesso em: 23 mar 2019.

10. Koller SH, Couto MCP, Von Hohendorff J. Manual de produção cientifica [Internet]. Porto Alegre: Penso Editora. 2014. Disponivel em: https://books.google.com.br/books?hl=p-

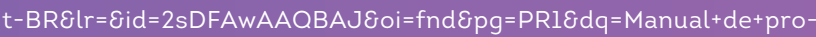
du\%C3\%A7\%C3\%A3o+cient\%C3\%ADfica\&ots=yFpwM1ZwOPEsig=NHEKhFn5ebvockm5-wwcLeGglKU\#v=onepage\&q=Manual\%20de\%20 produ\%C3\%A7\%C3\%A3○\%20cient\%C3\%ADfica\&f=false. Acesso em: 06 mai 2019.

11. Ursi ES, Galvão CM. Prevenção de lesões de pele no perioperatório: revisão integrativa da literatura. Rev. Latino Americana de Enfermagem [Internet]. 2006 [cited 2018 Nov 25]; 14(1):124-31. Available from: http://www. scielo.br/pdf/rlae/v14nl/v14nlal7.pdf. Acesso em: 08 abr 2019.

12. Souza MTD, Silva MDD, Carvalho RD. Revisão integrativa: o que é e como fazer. Revista Einstein [Internet]. 2010; 8(1): 102-106. Disponivel em: https:// www.scielo.br/pdf/eins/v8nl/pt_1679-4508-eins-8-1-0102. Acesso em: 06 mai 2019.

13. Araújo MIR, Moreira ACA, Silva MJD, Aragão AEDA, Freitas CASL, Monteiro PAA. Sexualidade e Envelhecimento: Necessidades Identificadas para a Construção de uma Tecnologia Educacional. Revista de Enfermagem UFPE / Revista de Enfermagem UFPE [Internet]. 2017; 11(7): 2674-82. Disponivel em: https://periodicos.ufpe.br/revistas/revistaenfermagem/article/ view/23439/19138. Acesso em: 19 fev 2019.

14. Goh AM, Loi SM, Westphal A, Lautenschlager NT. Person-centered care and engagement via technology of residents with dementia in aged care facilities. International psychogeriatrics [Internet]. 2017; 29(12): 2099-2103. Disponivel em: https://www.cambridge.org/core/journals/international-psychogeriatrics/article/personcentered-care-and-engagement-via-technology-of-residents-with-dementia-in-aged-care-facilities/15F22D1BA58FB5BB695B3969063EA3D4. Acesso em: 22 fev 2019.

15. Landeiro MJL, Peres HHC, Martins TV. Evaluation of the educational technology" Caring for dependent people" by family caregivers in changes and transfers of patients and tube feeding. Revista latino-americana de enfermagem [Internet]. 2016; 24. Disponivel em : http://www.scielo.br/pdf/ rlae/v24/pt_0104-1169-rlae-24-02774.pdf. Acesso em: 19 fev 2019.

16. Goes TM, Polaro SHI, Gonçalves LHT. Cultivo do bem viver das pessoas 
idosas e tecnologia cuidativo-educacional de enfermagem. Enferm Foco [Internet]. 2016; 7(2): 47-51. Disponivel em: http://revista.cofen.gov.br/index.php/enfermagem/article/view/794/319. Acesso em: 19 fev 2019.

17. Chiu CJ, Hu YH, Lin DC, Chang FY, Chang CS, Lai CF. The attitudes, impact, and learning needs of older adults using apps on touchscreen mobile devices: Results from a pilot study. Computers in Human Behavior [Internet]. 2016; 63: 189-97. Disponivel em: https://www.sciencedirect.com/ science/article/pii/S0747563216303478. Acesso em: 18 fev 2019.

18. Barros EJL, Santos SSC, Gomes GC, Erdmann AL. Gerontotecnologia educativa voltada ao idoso estomizado à luz da complexidade. Revista Gaúcha de Enfermagem [Internet]. 2012; 33(2): 95-101. Disponivel em: http://www.scielo.br/pdf/rgenf/v33n2/14.pdf. Acesso em: 19 fev 2019.

19. Nietsche EA, Teixeira E, Medeiros HP. Tecnologias cuidativo-educacionais: Uma possibilidade para o empoderamento do (a) enfermeiro(a). Porto Alegre: Moriá, 2014

20. Áfio ACE, Balbino AC, Alves MDS, Carvalho LVD, Santos MCL, Oliveira NR. Análise do conceito de tecnologia educacional em enfermagem aplicada ao paciente. Revista da Rede de Enfermagem do Nordeste [Internet]. 2014; 15(1): 158-65. Disponivel em: http://periodicos.ufc.br/rene/article/ view/3108/2382. Acesso em: 23 mar 2019.

21. Razera APR, Buetto LS, Lenza NDFB, Sonobe HM. Video educativo: estratégia de ensino-aprendizagem para pacientes em tratamento quimioterápico. Cienc Cuid Saude [Internet]. 2013; 13(1): 172-7. Disponivel em:http://periodicos.uem.br/ojs/index.php/CiencCuidSaude/article/ view/19659/pdf_156. Acesso em: 23 mar 2019.

22. Fernandes AFC. Construção de um video educativo sobre detecção precoce do câncer de mama. Revista Brasileira de Cancerologia [Internet]. 2013: 59(3): 401-7. Disponivel em: http://wwwl.inca.gov.br/rbc/n_59/ v03/pdf/10-artigo-construcao-video-educativo-sobre-deteccao-precoce-cancer-mama.pdf. Acesso em: 08 abr 2019.

23. Fleming SE, Reynolds J, Wallace B. Lights... camera... action! a guide for creating a DVD/video. Nurse Educator [Internet]. 2009; 34(3): 11821. Disponivel em: https://journals.lww.com/nurseeducatoronline/Abstract/2009/05000/Lights_-__Camera__-_Action__A_Guide_for_Creating_a.14.aspx. Acesso em: 28 fev 2019.

24. Duarte UO. Desenvolvimento e validação de tecnologia educativa leve-dura, sob o formato de aplicativo multimídia para plataforma móvel, para favorecimento a garantia do dever de cuidado com idoso no município de Mossoró-RN. 2018. Dissertação (Mestrado em Cognição, Tecnologias e Instituições) - Universidade Federal Rural do Semi-Árido, Mossoró-RN, 2018. Disponivel em: http://repositorio.ufersa.edu.br/bitstream/ prefix/1099/1/Uliss\%c3\%a9aOD_DISSERT.pdf. Acesso em: 28 fev 2019.

25. Estai M, Kanagasingam Y, Xiao D, Vignarajan J, Huang B, Kruger E et al. A proof-of-concept evaluation of a cloud-based store-and-forward telemedicine app for screening for oral diseases. Journal of telemedicine and telecare [Internet]. 2016; 22(6): 319-25. Disponivel em: https://www.ncbi. nlm.nih.gov/pubmed/26377126. Acesso em: 08 abr 2019.

26. Batista EB, Silva LWS, Moura LR, Queiroz VAR, Matos RS, Silva SJL et al. Inclusão Digital como ferramenta ao envelhecimento ativo: um relato de experiência. Prisma.com [Internet]. 2019; 38: 69-81. Disponivel em : https://ojs.letras.up.pt/ojs/index.php/prismacom/article/view/5428. Acesso em: 15 abr 2019
27. Martínez-Alcalá CI, Rosales-Lagarde A, Alonso-Lavernia MDLÁ, Ramirez-Salvador JÁ, Jiménez-Rodriguez B, Cepeda-Rebollar RM et al. Inclusão digital em idosos: uma comparação entre oficinas de alfabetização digital presencial e mista. Fronteiras em TIC [Internet]. 2018; 5:21. Disponivel em : https://www.frontiersin.org/articles/10.3389/fict.2018.00021/full. Acesso em: 08 abr 2019

28. Pereira ELC, Sanguino GZ, Ronchi TS, Previato GF, Jaques AE, Baldissera VDA. Tecnologias educativas gerontogeriátricas nas diferentes temáticas de saúde: uma revisão integrativa. Revista de Enfermagem do Centro Oeste Mineiro [Internet]. 2019; 9: 1-8. Disponivel em: http://seer.ufsj.edu.br/index. php/recom/article/view/2768. Acesso em 19 fev 2019

29. Berardinelli LMM, Guedes NAC, Ramos JP, Nascimento MG. Tecnologia educacional como estratégia de empoderamento de pessoas com enfermidades crônicas [Educational technology as a strategy for the empowerment of people with chronic illnesses]. Revista Enfermagem UERJ [Internet]. 2014; 22(5): 603-9. Disponivel em: http://www.facenf.uerj.br/v22n5/ v22n5a04.pdf. Acesso em: 28 fev 2019.

30. Cordeiro LI, Lopes TDO, Lira LEDA, Feitoza SMDS, Bessa MEP, Pereira MLD et al. Validation of educational booklet for HIV/Aids prevention in older adults. Revista brasileira de Enfermagem [Internet]. 2017; 70(4): 77582. Disponivel em:http://www.scielo.br/scielo.php?script=sci_arttextEpi$d=$ S0034-71672017000400775\&lng=en\&tlng=en. Acesso em: 23 mar 2019.

31. Camacho ACLF, Abreu LTA, Leite BS, Mata ACO, Louredo DS, Silva RP. Validação de cartilha informativa sobre idoso demenciado pelos enfermeirose acadêmicos de enfermagem: estudo observacional-transversal. Revista de Pesquisa Cuidado é Fundamental Online [Internet]. 2014; 6(1): 8-16. Disponivel em: https://www.redalyc.org/articulo.oa?id=5057/5057506203_210. Acesso em: 23 mar 2019.

32. Arruda LM, Avansi TA. Analfabetismo na terceira idade: pesquisa do analfabetismo em Sinop-MT. Eventos Pedagógicos [Internet]. 2014; 5(2): 435-42. Disponivel em: http://sinop.unemat.br/projetos/revista/index.php/ eventos/article/view/1425. Acesso em: 06 mai 2019.

33. Santos LTM, Bastos MG. Desenvolvimento de material educacional sobre doença renal crônica utilizando as melhores práticas em letramento em saúde. Brazilian Journal of Nephrology [Internet]. 2017; 39(1): 55-58. Disponivel em: https://www.scielo.br/scielo.php?pid=S0101-28002017000100 055\&script=sci_arttext\&tlng=pt. Acesso em: 23 mar2019.

34. Cruz FOAM, Ferreira EB, Vasques CI, Mata LRF, Reis PED. Validação de manual educativo para pacientes com câncer de cabeça e pescoço submetidos à radioterapia. Revista Latino-Americana de Enfermagem [Internet]. 2016; 24: 1-9. Disponivel em: http://www.scielo.br/pdf/rlae/v24/pt_ 0104-1169-rlae-24-02706.pdf. Acesso em: 28 fev 2019.

35. Chininthorn P, Glaser M, Tucker WD, Diehl JC. Exploration of deaf people's health information sources and techniques for information delivery in Cape Town: a qualitative study for the design and development of a mobile health app. JMIR human factors [Internet]. 2016; 3(2): e28. Disponivel em: https://www.ncbi.nlm.nih.gov/pubmed/27836819. Acesso em: 08 abr 2019.

36. Módolo CM. Infográficos: caracteristicas, conceitos e principios básicos. In: Congresso Brasileiro de Ciências da Comunicação da Região Sudeste [Internet]. 2007; 12:1-15. Disponivel em: http://www.intercom. org.br/papers/regionais/sudeste2007/resumos/r0586-1.pdf. Acesso em: 06 mai 2019 